\title{
Error Analysis of Deep Sequencing of Phage Libraries: Peptides Censored in Sequencing
}

\author{
Wadim L. Matochko and Ratmir Derda \\ Department of Chemistry and Alberta Glycomics Centre, University of Alberta, Edmonton, AB, Canada T6G 2 G2 \\ Correspondence should be addressed to Ratmir Derda; ratmir@ualberta.ca
}

Received 6 May 2013; Accepted 30 July 2013

Academic Editor: Yanxin Huang

Copyright ( 2013 W. L. Matochko and R. Derda. This is an open access article distributed under the Creative Commons Attribution License, which permits unrestricted use, distribution, and reproduction in any medium, provided the original work is properly cited.

\begin{abstract}
Next-generation sequencing techniques empower selection of ligands from phage-display libraries because they can detect low abundant clones and quantify changes in the copy numbers of clones without excessive selection rounds. Identification of errors in deep sequencing data is the most critical step in this process because these techniques have error rates $>1 \%$. Mechanisms that yield errors in Illumina and other techniques have been proposed, but no reports to date describe error analysis in phage libraries. Our paper focuses on error analysis of 7-mer peptide libraries sequenced by Illumina method. Low theoretical complexity of this phage library, as compared to complexity of long genetic reads and genomes, allowed us to describe this library using convenient linear vector and operator framework. We describe a phage library as $\mathrm{N} \times 1$ frequency vector $n=\left\|\mathrm{n}_{i}\right\|$, where $\mathrm{n}_{i}$ is the copy number of the $i$ th sequence and $\mathrm{N}$ is the theoretical diversity, that is, the total number of all possible sequences. Any manipulation to the library is an operator acting on $n$. Selection, amplification, or sequencing could be described as a product of a $\mathrm{N} \times \mathrm{N}$ matrix and a stochastic sampling operator $(\mathbf{S a})$. The latter is a random diagonal matrix that describes sampling of a library. In this paper, we focus on the properties of Sa and use them to define the sequencing operator (Seq). Sequencing without any bias and errors is $\mathbf{S e q}=\mathbf{S a} \mathrm{I}_{\mathrm{N}}$, where $\mathrm{I}_{\mathrm{N}}$ is a $\mathrm{N} \times \mathrm{N}$ unity matrix. Any bias in sequencing changes $\mathrm{I}_{\mathrm{N}}$ to a nonunity matrix. We identified a diagonal censorship matrix (CEN), which describes elimination or statistically significant downsampling, of specific reads during the sequencing process.
\end{abstract}

\section{Introduction}

In vitro selection experiments-such as phage display $[1,2]$, RNA display, SELEX, and DNA aptamer selection $[3,4]-$ employ large libraries, from which $10^{2}-10^{6}$ active sequences are identified through iterative rounds of selection and amplification. With the recent emergence of deep sequencing, it became possible to extract a large amount of information from the libraries before and after selection [5-10]. Deep examination of the library is a promising technique for direct evaluation of binding capacities of all binding sequences from one panning experiment. Deep sequencing also allows the characterization of unwanted phenomena in selection, such as amplification bias $[6,11]$.

Analysis of $10^{6}$ reads by deep sequencing gave rise to a large number of errors that were not present in the analysis based on the small number of sequences obtained using the Sanger method. Analysis of errors in information-rich datasets is a problem with over 50 years of history; correction of digital data made of bits or words is a topic of intense research in communication theory [12]. As phage display operates with limited digital sets, data analysis techniques from the communication theory could be applied to phage display. For example, Rodi and coworkers used a positional frequency matrix to calculate the informational content or Shannon entropy of each sequence [13]. This approach could be used to distinguish potential fast growing sequences from potential hits [14]. With the introduction of deep sequencing, the problem of error analysis in phage display becomes identical to a classical information theory problem: "reproducing at one point, either exactly or approximately, a message selected at another point" [15]. The "message" is the sequence information stored in the library. Sequencing process transmits this information and makes either stochastic or predictable errors. Understanding the sources of errors during sequencing could provide mechanisms for bypassing 
them, for correcting the errors, and for maximizing the amount of useful information received from sequencing.

There are over 10,000 published literature reports that contain the terms "deep sequencing" or "next generation sequencing" or any of the trademark names such as "Illumina" (reference: ISI database). Among these reports, less than 10 published reports describe sequencing of phagedisplayed libraries [5-7, 9, 10, 16-19]. Deep sequencing efforts in the literature are largely focused on genome assembly and metagenomic analyses. The error analysis techniques tailored for genome assembly cannot be used directly for analysis of phage libraries because the data output from phage library sequencing is very different from the genome assembly. In genome assembly, genomic DNA is shredded into random fragments and sequenced. The genome is then assembled from these fragments in silico. Although multiple fragments cover each area of the genome, the probability to observe two identically shredded fragments is very small. Two exact sequences, thus, could be considered amplification artifacts and removed by error analysis software. On the contrary, in phage-display sequencing, the reads are exactly of the same length. Duplication of the same read is important for validation of the accuracy of this read. Some researchers focus exclusively on reads that have been observed multiple times and discard singleton reads as erroneous [5]. Within each library, the copy numbers of sequences range continuously by six or more orders of magnitude $[5,6,9]$. Some phage clones are observed in the entire library only a few times; other clones could be present at copy number of 100,000 per sequencing run $[5,6,9]$. Unlike multiple cells with identical genomes, each screen is unique: identical set of sequences with identical copy numbers cannot be obtained even if the screen is repeated due to stochastic number of the screen that contains low copy number of binding clones [20].

Metagenomic analyses of microorganisms recovered from environmental samples [21, 22], also known as "microbiome" [23] and "viriome" analyses [24], encountered similar problems to those observed in phage library analysis: the concentration of species observed in a particular sample is unequal [25]. The abundance of species might range by a few orders of magnitude [26]. It is possible that error analysis tools developed in the above areas could find use in phage display sequencing. For example, there are multiple published algorithms for removing errors from low copy number reads to ascertain that low copy number sequences are new species and not sequencing errors (e.g., see [2729] and references within). Metagenomic analysis is usually more complex than analysis of phage-display libraries. First, in metagenomics, the bacterial or viral genes must be assembled from short reads de novo. Second, there is no simple relationship between phylogenetic classification of "species" and the observed DNA sequence. Third, the exact number of species in the environment is unknown. On the other hand, sequencing of phage-displayed peptide libraries has none of these problems: (i) it requires no assembly steps because each sequence is covered by one read; (ii) a unique DNA sequence defines a unique "species"; and (iii) the theoretical complexity in synthetic libraries is known exactly. For small libraries, such as the library of 7-mer peptides, the complexity, $(20)^{7}$, is within the reach of next-generation sequencing. We see phage-displayed peptide libraries as an ideal model playground for the development of optimal error analysis and error correction protocols. It is possible that error analysis developed from phage libraries analysis could then be used in other areas such as genomic and metagenomic analyses.

The errors in sequencing could be divided into "annotated" and "invisible." The "annotated" errors that originate from misincorporation of nucleotides are annotated using Phred quality score [30]. These annotated errors are removed during the processing (see below). Examples of "invisible" errors are sequence-specific frame shifts that lead to emergence of truncated reads during the Illumina sequencing [31]. Invisible errors could also originate during the preparation of the libraries for sequencing. Examples are removal of AT-rich fragments during purification of dsDNA [32] and erroneous incorporation of nucleotides during PCR [33,34]. Mutations have the most significant impact on the observed diversity of the library. There are 63 ways to misspell a 21-mer-nucleotide sequence with a one-letter error (point mutation). The large dynamic range in concentrations of clones in the phage library exacerbates the problem. Clones that are present in high abundance $-10^{5}$ copies per read-are more prone to yield errors [6]. For example, we observed that random point mutations convert several short sequence with a copy number of $10^{5}$ to a library of sequences with copy numbers ranging from 1 to $10^{2}$ [11]. In attempt to unify error analysis into one convenient theoretical framework, we generalized all errors as follows. All errors either lead to disappearance of particular sequence or its conversion to another sequence of the same length. Errors, thus, operate within a finite sequence space, and it should be possible to use elementary linear algebra to generalize most processes that lead to errors.

\section{Theoretical Description}

See Table 1.

2.1. Operator Description of the Phage-Display Library and Selection Process. In our previous reports, we described the phage library as a multiset, or a set in which members can appear more than once [35]. This description also simplifies the analysis of the errors in these libraries. The multiset description represents a library with $\mathrm{N}$ theoretical members as an ordered set of $\mathrm{N}$ sequences and $\mathrm{N} \times 1$ copy number vector $(n)$ with positive integer copy numbers (Figure 1(a)). Any manipulation of a phage library-such as erroneous reading or selection-changes the numbers within the copy number vector. All manipulations to the multiset, thus, could be described by operators $(\mathbf{O p})$ that convert vector $n_{1}$ to another vector $n_{2}$ as $n_{2}=\mathbf{O p} n_{1}$ (Figure $1(\mathrm{c})$ ). For an $\mathrm{N} \times 1$ vector, the operator is $\mathrm{N} \times \mathrm{N}$ matrix. If elements are selected or eliminated independently of one another, the $\mathrm{N} \times \mathrm{N}$ matrix is diagonal (Figure $1(\mathrm{~d})$ ). This approach is uniquely convenient for libraries of short reads. For example, a library of 7-mers contains exactly $20^{7}=1.28 \times 10^{9}$ peptides and is described 
TABLE 1: Symbols and definitions used in the theoretical description section.

\begin{tabular}{|c|c|}
\hline Symbols & Meaning \\
\hline$A, a, f, m, n, k$ & Unless specified otherwise, normal font designates scalars \\
\hline$A, a, N, P,{ }^{1} \mathrm{n},{ }^{13} \mathrm{n}$ & Italic font designates vectors. Different vectors can be distinguished by the left-superscript notation \\
\hline A, a, Abc, Pan, Sa & Bold font designates operators or matrices (here all operators are matrices) \\
\hline${ }^{1} \mathrm{~A},{ }^{\mathrm{f}} \mathrm{Sa},{ }^{0.9} \mathrm{Sa},{ }^{0.5} \mathrm{Sa}$ & $\begin{array}{l}\text { Operators can be distinguished by the left-superscript notation. For sampling operator Sa, this } \\
\text { notation specifies the sampling fraction of the Sa operator }\end{array}$ \\
\hline $\mathrm{A}_{1}, \mathrm{a}_{2}, \mathrm{~A}_{i}, \mathrm{a}_{j}$ & Normal font with right subscript designates scalar values of the vector \\
\hline$A_{11}, A_{21}, A_{i j}, A_{i i}$ & Normal font with two right subscripts designates scalar values of the 2D matrix \\
\hline$\left\|A_{1} \ldots A_{5}\right\|$ & Description of the scalar elements in the vector \\
\hline$\left\|\mathrm{A}_{\mathrm{ij}} \ldots \mathrm{A}_{\mathrm{ii}}\right\|$ & Description of the scalar elements in the matrix \\
\hline $\mathrm{x} \in[\mathrm{A} B]$ & Scalar $\mathrm{x}$ belongs to the inclusive scalar interval $[\mathrm{A} \mathrm{B}]$; that is, $\mathrm{A} \leq \mathrm{x} \leq \mathrm{B}$ \\
\hline$x \in[A B]$ & Vector $x$ belongs to the "vector interval" $[A B]$; that is, for every element $A_{i} \leq \mathrm{x}_{\mathrm{i}} \leq \mathrm{B}_{\mathrm{i}}$ \\
\hline$\{\mathrm{A} \mathrm{B} \mathrm{C} \mathrm{\ldots X}\}$ & Set where $\mathrm{A}, \mathrm{B}, \mathrm{C}, \ldots, \mathrm{X}$ are the unique elements of the set \\
\hline$\{\mathrm{A}(\mathrm{a}) \mathrm{B}(\mathrm{b}) \ldots \mathrm{X}(\mathrm{x})\}$ & $\begin{array}{l}\text { Multiset (2-tuple) where A, B,.., X are the unique elements and a, b, } \mathrm{x} \text { are the scalars describing the } \\
\text { copy numbers of the A, B, X elements }\end{array}$ \\
\hline $\mathrm{I}_{\mathrm{N}}$ & Unity matrix of the Nth order; that is, $\mathrm{N} \times \mathrm{N}$ matrix $\left\|\mathrm{A}_{\mathrm{ij}}\right\|, \mathrm{A}_{\mathrm{ij}}=\delta_{\mathrm{ij}}$ (Kronecker delta) \\
\hline
\end{tabular}

completely using a $10^{9}$-element vector. This size is accessible to the computational capacity of most desktop computers.

In operator notation, phage display can be described as

$$
\text { Sel = Pan Naive, }
$$

where Naive is the copy number vector for naïve library, $\mathrm{Sel}$ is the copy number vector after panning, and Pan is a panning operator. In standard phage display, the Pan operator is a complex product of all manipulation steps (binding, amplification, dilutions, etc.). If a screen uses no amplification and uses deep-sequencing $[9,16]$, or large-scale Sanger sequencing $[36,37]$ to analyze the enrichment, it might be possible to define the panning process as a simple product of two operators as follows:

$$
\begin{gathered}
\text { Pan }={ }^{\mathrm{f}} \text { Sa } \mathbf{K}_{\mathbf{a}}, \\
\text { Sel }={ }^{\mathrm{f}} \text { Sa } \mathbf{K}_{\mathbf{a}} \text { Naive, }
\end{gathered}
$$

where $\mathbf{K}_{\mathbf{a}}$ is a deterministic "association" operator, which contains association constants for every phage clone present in the library. Description of such operator is beyond the scope of this paper and we recommend consulting other reports that attempted to generalize the selection procedure [20]. Another operator in (3) is a sampling operator ( $\left.{ }^{\mathrm{f}} \mathbf{S a}\right)$, which describes stochastic sampling of the library with $\mathrm{m}$ sequences to yield a sublibrary with $\mathrm{f}^{*} \mathrm{~m}$-sequences, where $\mathrm{f} \epsilon$ $\left[\begin{array}{ll}0 & 1\end{array}\right]$ is a sampling fraction. ${ }^{\mathrm{f}} \mathbf{S a}$ operator has the following properties, which emanate from physical properties of the sampling procedure:

(I)

${ }^{\mathrm{f}} \mathbf{S a}_{\mathrm{i}} 0=0$ (sampling does not create new sequences from nonexisting sequences).
(II) ${ }^{\mathrm{f}} \mathrm{Sa}$ is a diagonal operator with diagonal scalar functions $\left\|\mathrm{Sa}_{11} \mathrm{Sa}_{22} \cdots \mathrm{Sa}_{\mathrm{NN}}\right\|, \mathrm{Sa}_{\mathrm{i}}(0)=0$.

(III) In $B={ }^{\mathrm{f}} \mathbf{S a} A, B$ is a vector of positive integers, $\mathrm{B}_{i} \geq 0$ and $\operatorname{sum}(B)=\mathrm{f}^{*} \operatorname{sum}(A)$. Integer values ensure that the observable values of the operator have physical meaning. The clone could be observed once (1), multiple times $(2,3$, etc.), or not observed at all (0).

(IV) Sa is nondeterministic operator. When applied to the same vector, the operator does not yield the same result but one of the possible vectors that satisfy rules (I-III). The majority of the solutions of the operator, however, reside within a deterministic confidence interval ${ }^{\mathrm{f}} \mathrm{Sa} A \in\left[{ }^{\mathrm{lo}} \mathrm{C} \quad{ }^{\text {hi }} \mathrm{C}\right]$.

(V) As a consequence from (IV), operator Sa is nonlinear, noncommutative, and nondistributive.

(VI) Large sum of sampling operators with same f should "average out" to yield $\mathrm{I}_{\mathrm{N}}$ unity matrix

$$
\begin{array}{r}
\frac{\left({ }^{\mathrm{f}} \mathbf{S a}_{1}+{ }^{\mathrm{f}} \mathbf{S a}_{2}+{ }^{\mathrm{f}} \mathbf{S} \mathbf{a}_{3}+\cdots{ }^{\mathrm{f}} \mathbf{S a}_{\mathrm{k}}\right)}{\mathrm{k}} \longrightarrow \mathrm{f}^{*} \mathrm{I}_{\mathrm{N}}, \\
\text { as } \mathrm{k} \longrightarrow \infty .
\end{array}
$$

The Sa operator is simple to implement as a random array indexing function in any programming language (e.g., see Supplementary Schemes S1, and S2 available online at http://dx.doi.org/10.1155/2013/491612). It might be possible to express ${ }^{\mathrm{f}} \mathrm{Sa}$ analytically for any $\mathrm{f}$ as a diagonal matrix (Figure 1(d)). In this paper, we use numerical treatment by an array sampling function because it is more convenient for multisets of general structure. We tested the random indexing implementation to show that the sampling algorithm yields a normal distribution for a large number of samples (Supplementary Figure S1). Despite the simplicity of ${ }^{\mathrm{f}} \mathbf{S a}$ 


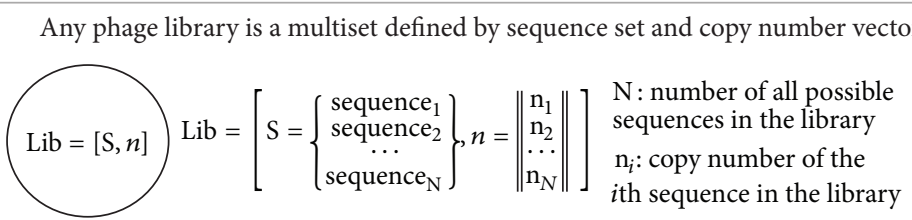

(a)

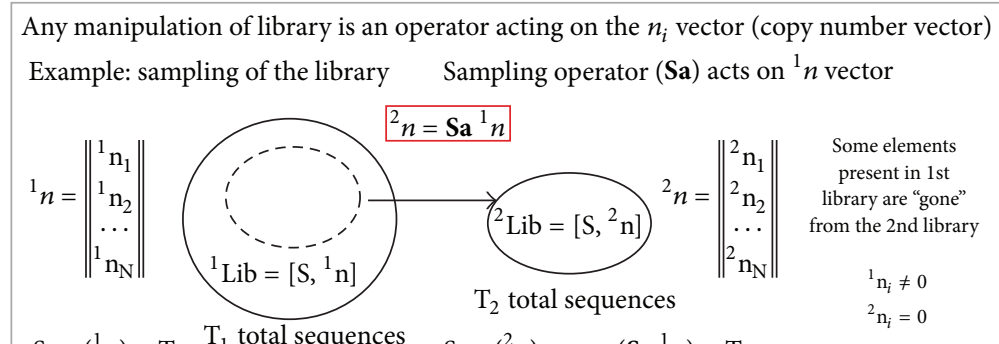

$\operatorname{Sum}\left({ }^{1} n\right)=\mathrm{T}_{1} \quad \mathrm{~T}_{1}$ total sequences $\quad \operatorname{Sum}\left({ }^{2} n\right)=\operatorname{sum}\left(\operatorname{Sa}^{1} n\right)=\mathrm{T}_{2}$

Total (sum) and unique sequences (uni)
$\operatorname{sum}(\mathrm{n})=\mathrm{n} \cdot|1|=\sum_{i=1}^{\mathrm{N}} \mathrm{n}_{i} \quad|1|=\left\|\begin{array}{c}1 \\ 1 \\ \cdots \\ 1\end{array}\right\|$
$\operatorname{uni}(\mathrm{n})=\sum_{i=1}^{\mathrm{N}} 1-\delta\left[\mathrm{n}_{i}\right] \quad \delta[\mathrm{n}]= \begin{cases}1, & \mathrm{n}=0 \text { Kronecker } \\ 0, & \mathrm{n} \neq 0 \text { delta }\end{cases}$

(b)

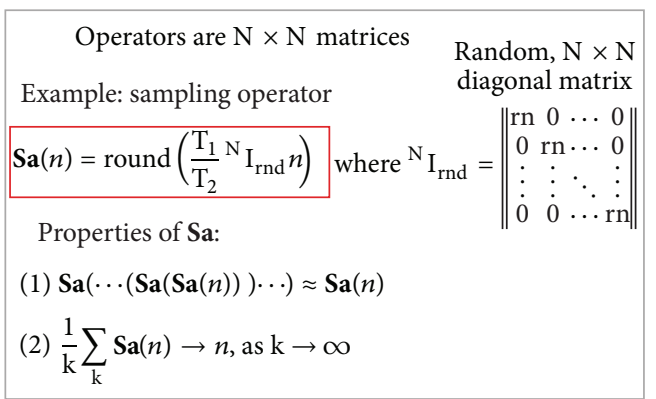

(d)

Multiset description of the phage display selection

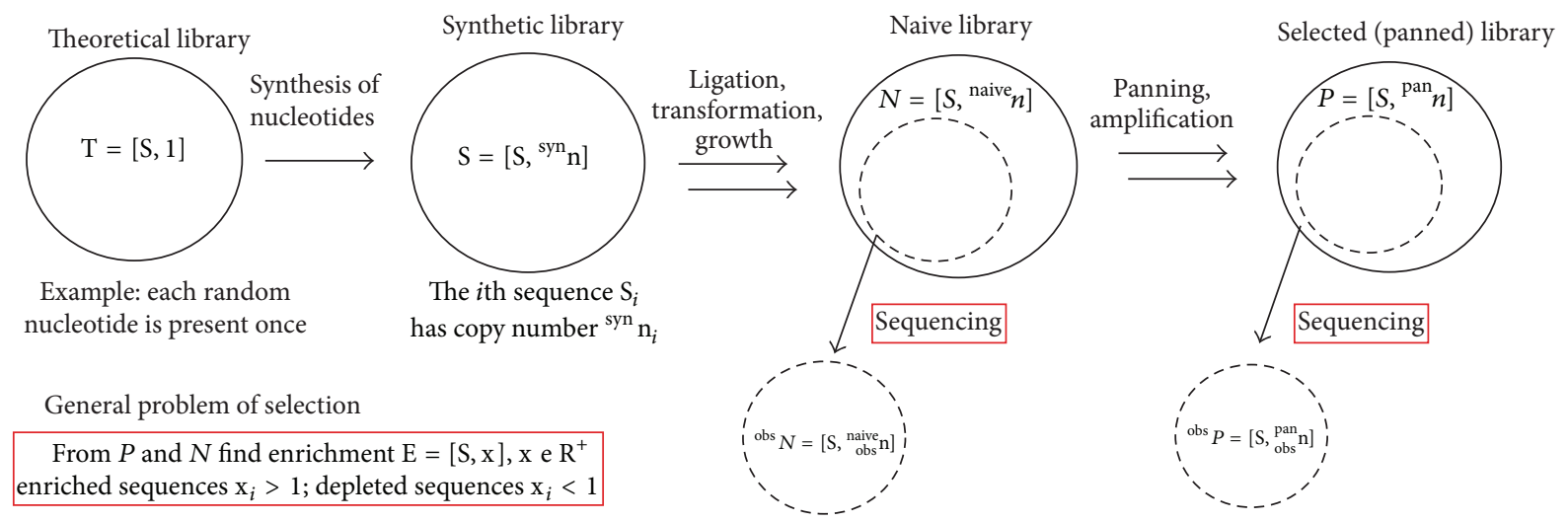

$P$ and $N$ cannot be measured. They have to be approximated from the "observables":

(e)

Figure 1: (a) Phage library can be described by multisets made of $S=\{$ sequence set $\}$ and $n=\|$ vector of copy numbers $\|$. Any change to the library can be described as function/operator acting on the $n$. (b) Relevant functions are calculations of total sequences (sum) and unique sequences (uni). (c) Any transformation of library to another library is an operator acting on $n$. Sampling of libraries to yield a sublibrary is the most important operator. (d) It can be described as $\mathrm{N} \times \mathrm{N}$ matrix. Specifically, Sa is a diagonal matrix of values derived from random distribution. Rounding function is necessary to ensure the physical meaning of the sampling results. Sa acting on the same vector yields one of many vectors that have the same number of total elements. As a consequence, Sa is nonlinear, nondistributive, and noncommutative operator. Average of many Sa operators is a scalar (dilution factor). (e) Any screen of any library can be described as operators acting on the copy number vectors of the naïve (or theoretical) library. Copy number vectors cannot be observed directly. They have to be measured through sequencing. As sequencing contains sampling process (Sa operator), the result of sequencing is nondeterministic. Sequencing yields one of many possible observed copy number vectors, none of which are equal to the real copy number vector.

implementation-the entire code is <30 lines in MatLabthe script allows rapid calculation of the results of ${ }^{\mathrm{f}} \mathbf{S a}$ for a multiset of reasonable size (several million sequences, Figures 4 and 5) on a desktop computer.

We evaluated the behaviors of ${ }^{0.5} \mathrm{Sa}$ for several multisets. The probability to observe a specific solution is described in Figure 3(b). Individual solutions can be represented as lines with nodes on $X Y$-plane, where each node represents one element of the multiset (Figures 3(d) and 3(e)). The most probable solutions reside near the "expected solution" (represented as dotted line), and the probability to observe a solution where many elements deviate from the probable solution is low (Figure 3(e)). Graphical representation of the solutions highlights that sampling could lead to deviation of the frequency of the individual elements of the multiset; for example, Figure 3(e) describes $>2$ fold deviation from 


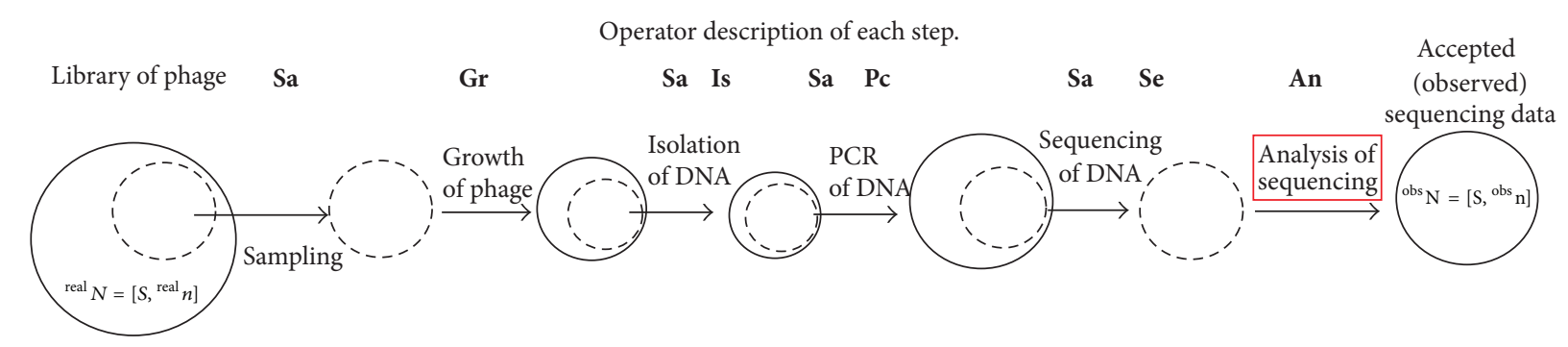

(a)

Relation between observed copy numbers and "real" copy numbers

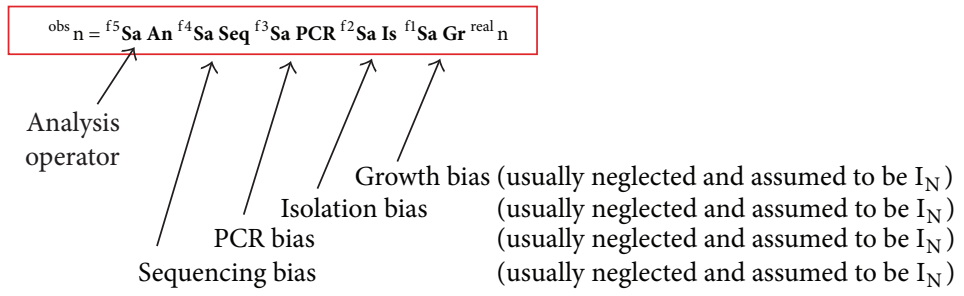

(b)
Analysis operator is a matrix that could compensate for problems and censorships caused by Se Pc Is Gr operators

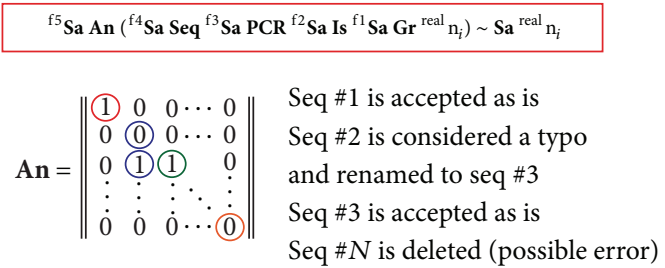

(c)

FIGURE 2: Operator description of the deep sequencing process. (a) A library of phage must be processed before deep sequencing. Each step involves sampling, which is either a deliberate partitioning of the sample or random loss of the sample. Each sample preparation state could (and does) introduce bias in sequence abundance. Each step, thus, is an operator chat changeing the $n$ vector. (b) If we ignore bias during preparation, operators could be approximated as unity vectors, and sequencing could be represented as a product of sampling and analysis operators. (c) Analysis operator (An) is a binary decision matrix, which describes what sequences are and are not considered as errors. Decisions, such as removal of sequences or correction of sequences, are the most important because they decide which "observed" sequences are considered "real." To make the analysis of the selection process meaningful, the same An operator should be used in all analyses.

the expected value for one of the elements. Figure 3(f) shows that the solution in which two elements deviate by $>2$ fold is improbable. This observation is a simple consequence of the multiplicity of the probabilities (large deviation from the average has probability $p$ and the probability to observe this deviation twice is $p^{2}$ ).

Even in small multisets, such as $\{\mathrm{A}(1) \mathrm{B}(2) \mathrm{C}(3) \mathrm{D}(4)\}$ made of four unique and 10 total elements, ${ }^{0.5} \mathrm{Sa}\{\mathrm{A}(1) \mathrm{B}(2) \mathrm{C}(3) \mathrm{D}(4)\}$ operation yields large number of solutions with equal probability, termed as redundant solutions (e.g., solutions that have equal probability in Figure 3(b)). Redundancy depends on the structure of the multiset (Figure S2). This redundancy makes the calculations of all probable solutions of Sa impractical. For sets even with 5-6 unique elements, identification of all vectors $B$, which satisfy equation $B={ }^{\mathrm{f}} \mathbf{S a} A$ and reside within a 95\% interval, requires hundreds of thousands of iterations (Figure S2 and S3). On the other hand, calculation of the confidence interval of each element $B_{1}$ of the vector $B$ converges rapidly. A multiset $\left\{\mathrm{A}_{1000}\right\}=\left\{\mathrm{A}_{1}(1) \mathrm{A}_{2}(2) \cdots \mathrm{A}_{1000}(1000)\right\}$ with 1000 unique elements and $1+2+3+\cdots+1000=500,500$ total elements is similar to an average deep sequencing data set (Figure 4). Calculation of all probable solutions of ${ }^{0.5} \mathrm{Sa}\left\{\mathrm{A}_{1000}\right\}$ is beyond the capabilities of most computers. However, the $99.9 \%$ confidence interval of all elements of vector $B={ }^{0.5} \mathrm{Sa}\left\{\mathrm{A}_{1000}\right\}$ can be calculated in $\sim 2$ minutes on an average desktop computer. The red dots in Figure 4 are ${ }^{\text {lo }} C_{i}$ and ${ }^{\text {hi }} C_{i}$ or the $99.9 \%$ high and low confidence interval of all elements $\mathrm{B}_{\mathrm{i}}$ (Figure 4 ).
The sampling operator is critical in phage display because sampling of libraries occurs in every step of the selection and the preparation of libraries for sequencing. The stochastic nature of sampling operators makes two identical screens "similar within a confidence interval." Solving (1) exactly is not possible, but it should be possible to estimate the solution within a confidence interval. Consider

$$
\text { Sel } \in\left[{ }^{\text {lo }} \mathbf{K}_{\mathbf{a}} \text { Naive; }{ }^{\text {hi }} \mathbf{K}_{\mathbf{a}} \text { Naive }\right] \text {, }
$$

where ${ }^{\text {lo }} \mathbf{K}_{\mathbf{a}}$ and ${ }^{\mathrm{hi}} \mathbf{K}_{\mathbf{a}}$ are diagonal matrices of the upper and lower confidence intervals for the association constants. A simulation of the behavior of the Sa operator (Figures 3 and S3) suggests that the relative sizes of the confidence intervals might be impractically large when the copy numbers of sequences are $<10$.

Multiple sampling events of the Sa operator yield a normal distribution for each element of the vector (Figure 3 ). Fitting this normal distribution could yield a "true" value of the process. This process is identical to the extrapolation of the average from the normal distribution of noisy data. Multiple algorithms for such extrapolation exist for one- and multidimensional stochastic processes $[38,39]$. We believe that $\mathrm{Sa}$ behaves as a one-dimensional stochastic process and it might be possible to extrapolate the true value of the sampling from 7 to 10 repeated instances of Sa (i.e., the number of data sufficient to fit an 1D normal distribution). The necessary practical steps towards solving (3) or (10) are the following. (i) Eliminate or account for any bias not related to binding (e.g., growth bias). (ii) Repeat the screen 


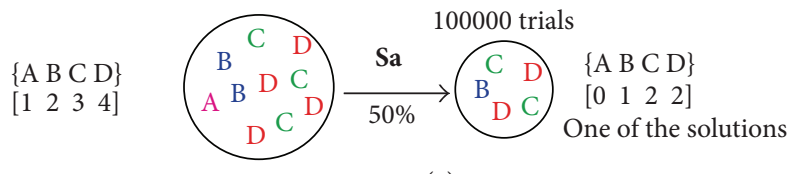

(a)

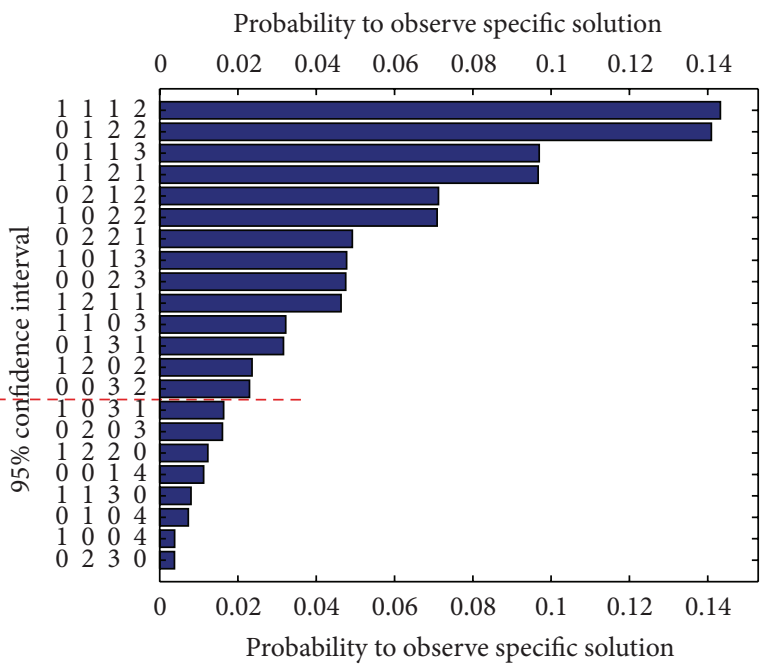

(b)

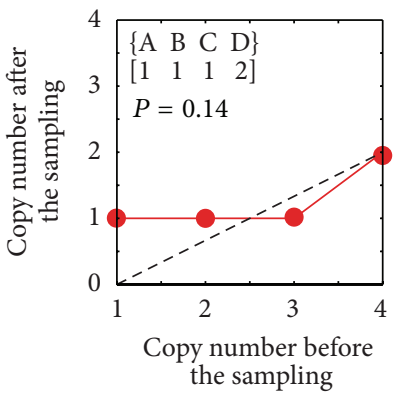

(c)

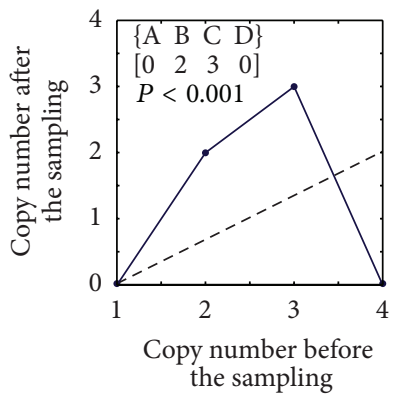

(e)

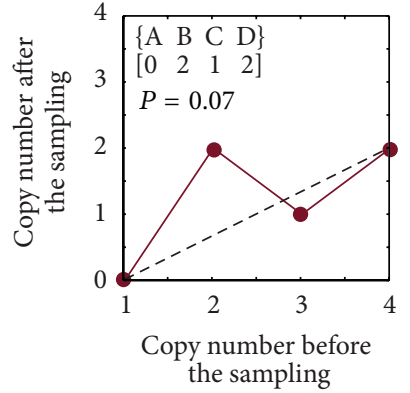

(d)

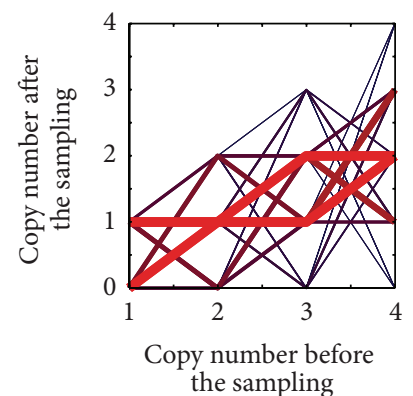

(f)

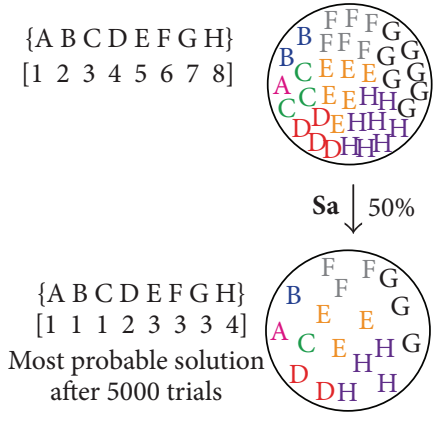

(g)

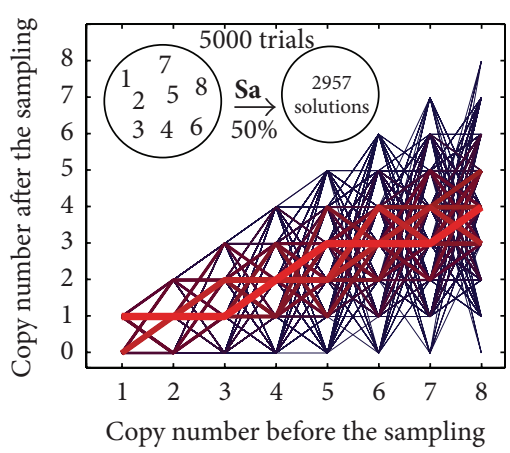

Copy number before the sampling

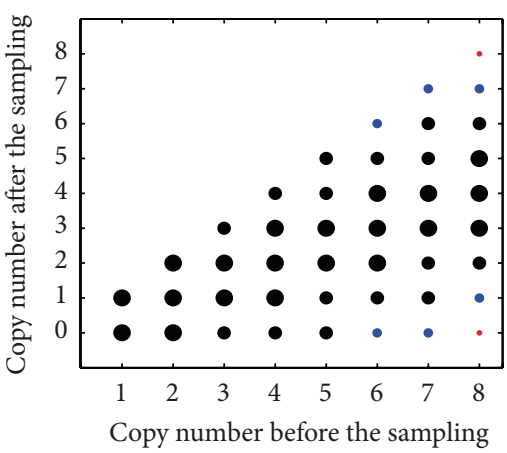

Number of data per point

- >1000 copies

- 100-1000 copies

- $10-100$ copies

- 1-10 copies

(h)

(i)

FIGURE 3: (a) Testing the sampling operator implemented as random indexing function using a model multiset. (b) In 100,000 trials, we observed 22 unique solutions from which 14 resided in a 95\% confidence interval. Solutions with 0 and 1 copies of element A were found at equal abundances ("redundant solutions"). (c) Representation of the most probable solution as a line with 4 nodes; " $p$ " is a probability to find the solution; dotted line is an expected "average solution" for 50\% sampling. (d) The 5th most probable solution; (e) least probable solution deviates the most form the average; (f) combination of all solutions. Red thick lines describe the most probable solutions; thin blue lines describe the least probable solutions. (g) Sampling of larger multisets yields more possible solutions (here, 2957 in 5000 trials). (h) All solutions of the sampling represented as lines. (i) Probability to observe a particular copy number after sampling. While (h) is the most accurate representations of the confidence intervals, the thin blue lines describe solutions outside the confidence interval; this representation is impractical due to large number of redundant solutions in larger multisets. In (i), confidence interval could be extrapolated from distributions of individual copy numbers (e): red dots are on or outside the confidence interval.

several times. (iii) Measure all copy numbers of all sequences, including zero values, with high confidence. Requirement (i) has been an ongoing effort in our group [11, 40] and other groups [13, 41-43]; for review see [11,44]. Deep sequencing makes it simple to satisfy requirement (ii) and obtain multiple instances of the same experiment. For example, we described the Illumina sequencing method that allows using barcoded primers to sequence 18 unrelated experiments in one deep 

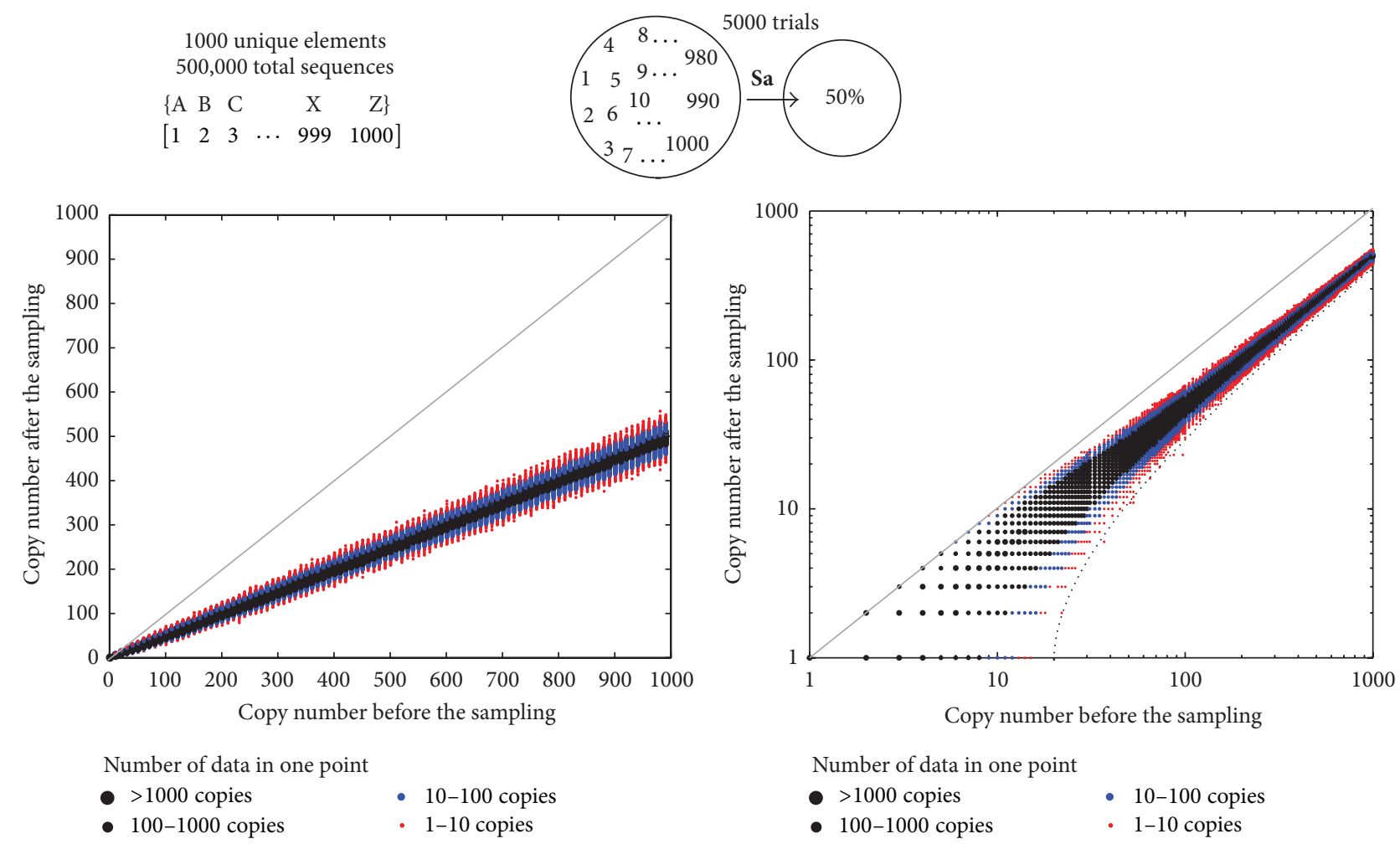

Figure 4: (a) Testing the sampling operator using a large multiset made of 1000 unique elements with 1000 different copy numbers. Images describe linear and log-scale representation of the confidence interval of the sampling operator. Solutions beyond this interval were not observed in 5000 trials. Dotted line represents an overestimate of the $99.9 \%$ confidence interval (for details, see Figure S4). Most probable outcomes of the Sa operator have either zero or one unique sequence beyond this interval. This line is used in subsequent sections (Figures 5 and 6). We note that distributions of the copy numbers have well-defined shape; according to central limit theorem, it is a normal distribution. With enough replicas, it should be possible to extrapolate the center of this distribution, define the solutions explicitly, and bypass the stochastic nature of the Sa operator.

sequencing experiment [45]. We recently scaled this effort to 50 primer sets and evaluated the performance replicas of simple selection procedures (in preparation).

The measurement of the copy numbers of sequences is a separate problem that can be described using the same sampling operators and bias operators that describe how the library is skewed by each preparation step. For example, isolation of DNA by gel purification disfavors ATrich sequences, whereas PCR favors sequence with within specific GC-content range [32]. The real sequence abundance in any phage library $\left({ }^{\text {real }} n\right.$ ), hence, has to be derived from the observed sequence abundance $\left({ }^{\text {obs }} n\right)$ by solving this equation:

$$
\begin{aligned}
{ }^{\text {obs }} n= & \left({ }^{\mathrm{f} 5} \text { Sa An }\right)\left({ }^{\mathrm{f} 4} \text { Sa Seq }\right)\left({ }^{\mathrm{f}} \mathbf{S a} \text { PCR }\right) \\
& \times\left({ }^{\mathrm{f} 2} \mathbf{S a} \mathbf{I s}\right)\left({ }^{\mathrm{f} 1} \mathbf{S a ~ G r}\right){ }^{\text {real }} n .
\end{aligned}
$$

In this equation, each operator in brackets describes a bias at a particular step. ${ }^{\mathrm{f}} \mathrm{Sa}$ describes sampling at that step, and f1f5 describe the sampling fractions. The bias in growth (Gr), isolation (Is), PCR amplification (PCR), and sequencing (Seq) could be related to the nucleotide sequences. The An analysis operator is a matrix that describes retaining, discarding, or correcting the sequence (Figure 2(b)). An ideal
An operator could compensate for the biases introduced by another operator (Figure 2(c)). To define such operator, (7) could be potentially solved using repeated sequencing of a well-defined model library. In the next applied section, we examine the real deep sequencing data and identify conditions under which these operators could be at least partially defined.

2.2. Analysis or the Error Cutoff in Deep Sequencing Reads. All next-generation sequencing techniques provide quality score (Phred Score) for every sequenced nucleotide. In Illumina sequencing, this score is related to the probability of the nucleotide being correct [46]. In low throughput Sanger sequencing, the Phred score monotonously decreases with read length and the mechanisms that yield errors in capillary electrophoresis are well understood. Common practice in Sanger sequencing is to discard all reads after the first nucleotide with a Phred score of 0 . In next-generation sequencing, the filtering of the reads is usually more stringent as follows.

(A) Discard reads that have at least one read that has score lower than "cutoff."

(B) Discard reads that had cumulative Phred score lower than cutoff. 


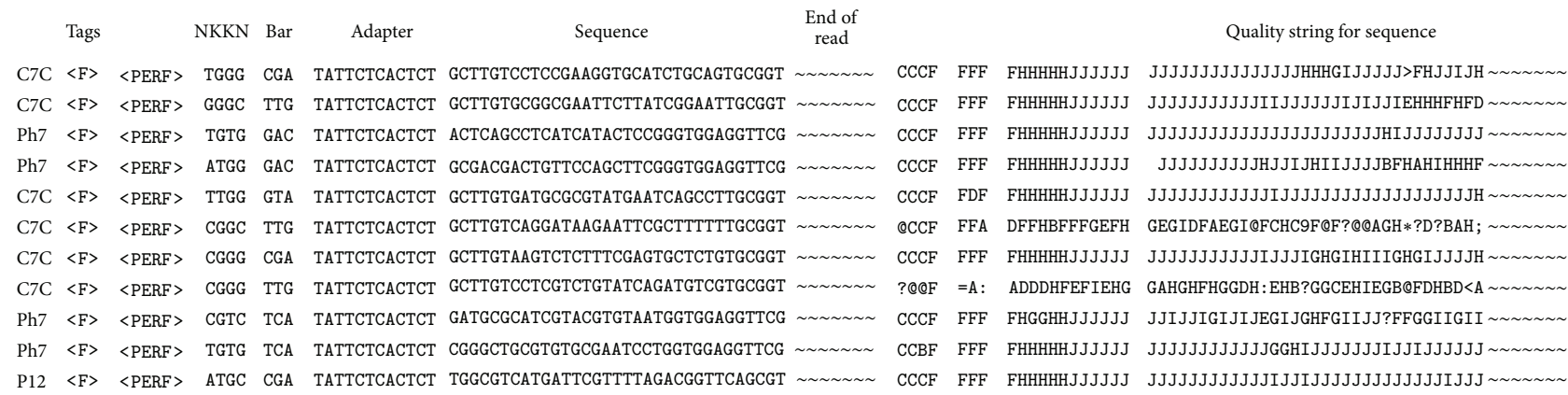

(a)

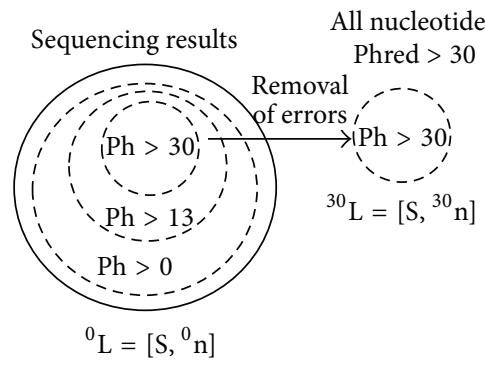

(b)

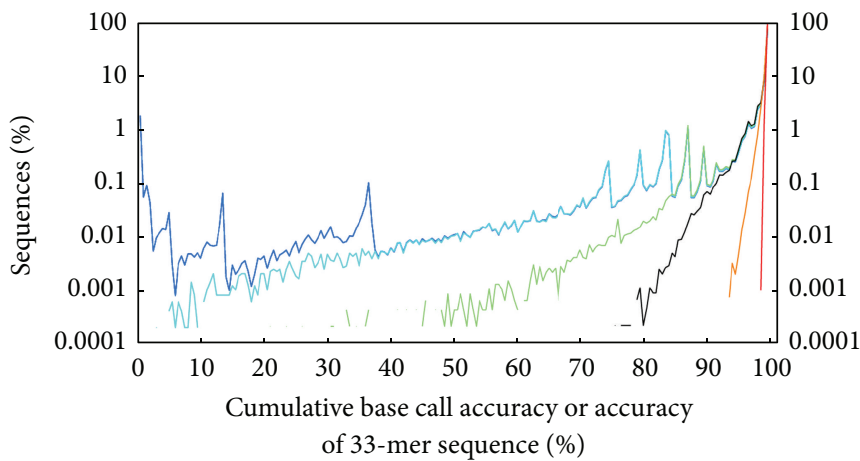

(d)
Phred score cutoff. Every nucleotide must have Phred score higher than:

\begin{tabular}{r|c|c|c|c|c|c|c|c}
\cline { 2 - 5 } & 1 & 5 & 8 & 13 & 20 & 30 & \multirow{2}{*}{$10^{\text {cutoff/10 }}$} \\
Base call accuracy (\%) & 21.0 & 68.0 & 84.0 & 95.0 & 99.0 & 99.9 & \\
(Base accuracy) $^{33}(\%)$ & 0.0 & 0.0 & 0.1 & 18 & 71.7 & 96.7 & \\
Mean 33-mer accuracy (\%) & 95.0 & 97.5 & 98.7 & 99.2 & 99.5 & 99.6 & $\prod_{i=1}^{33} 10^{\mathrm{Qi} / 10}$
\end{tabular}

(c)

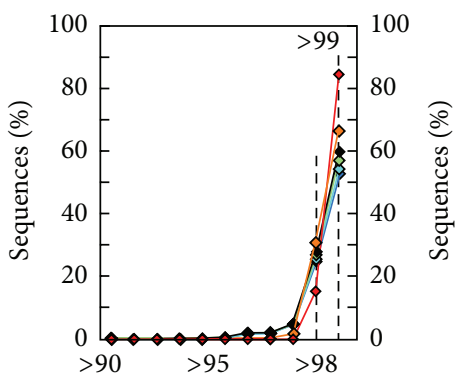

Cumulative base call accuracy or accuracy of 33-mer sequence (\%)

(e)

Figure 5: (a) Representative lines from the intermediate file from Illumina deep sequencing analysis (for more information see ERROR_TAG_data0001.txt in Section 4 and our previous publication [6]). The reads have been parsed to identify adapters and barcodes. Each read has been tagged according to the library type, direction of the read, and quality of the adapter regions. We use this intermediate library to identify reads that harbor erroneous nucleotides. (b) Multiset view of the intermediate library. The library contains subsets that have low, medium, and high quality reads. Error filtering of this intermediate library to eliminates any read with Phred score below 30 yields a high quality library of reads ${ }^{30} \mathrm{~L}$. (c) Mean accuracy of the reads in the library after error filtering ranges from $95 \%$ to $99.6 \%$. Even for very low-quality cutoff, Phred $>1$, the average read quality is $95 \%$. (d) Distribution of cumulative read accuracy in libraries processed using different cutoffs. (e) Linear plot of the data presented in (d) with zoom in on the region with $>90 \%$ cumulative accuracy.

(C) Use a combination of $\mathrm{A}$ and $\mathrm{B}$ (accept reads with minimal cutoff and minimal cumulative score).

Many of the error analyses in the area of deep sequencing are designed for genetic reads, which have variable lengths and unknown sequence throughout the whole read. Analysis of the reads in a phage-display library is a simpler problem because phage-derived constant adapter regions flank the variable reads. Identification of the adapter region is a necessary first step in the analysis. Reads, in which the adapters cannot be mapped, cannot be used. We designed algorithms to recover reads, even if adapters were hampered by truncation, deletion, or mutation [6]. We observed that the reads flanked by the erroneous adapters had a significantly higher error rate than reads flanked by "perfect" adapters (unpublished). Example of mapping of flawed reads in Illumina sequencing is provided in ERROR_TAG_data0001.txt (see Section 4). In the remaining sections, we analyze the population of the sequences preceded by a "perfect" adapter to identify possible sequence-specific biases.

We analyzed a typical library sequenced by Illumina using various cutoffs (Figure 5). We analyzed a 33-bp segment of the library that contained variable seven amino acids and a constant region and GGGS terminus. A simple cutoff that discards reads with Phred $<1$ nucleotides yields library 
termed ${ }^{1} n$, which had an average $95 \%$ accuracy of the 33-nucleotide read. Reads that do not contain Phred $=0$ nucleotide rarely contain multiple low-quality reads. The ${ }^{1} n$ library was bimodal: $80 \%$ of the reads had overall accuracy of $99 \%$, very few reads with accuracy 5-90\%, and significant number of reads with accuracy of $1 \%$ (Figures 5(d) and 5(e)). These observations suggest that reads can be divided into (i) reads free of errors and (ii) reads with multiple errors.

An example of a more stringent cutoff is elimination of reads with Phred $<13$ nucleotides; this process yielded a library ${ }^{13} n$ in which every nucleotide had $>95 \%$ confidence. The number of total reads in ${ }^{13} n$ was $10 \%$ less than number of reads in ${ }^{1} n$; that is, $\operatorname{sum}\left({ }^{13} n\right)=0.9 \operatorname{sum}\left({ }^{1} n\right)$. The observed average read accuracy of the read in the ${ }^{13} n$ library was $99.2 \%$. Theoretically, the 0.95 confidence cutoff in a 33mer nucleotide could yield reads with accuracy as low as $(0.95)^{33}=18 \%$. In practice, the probability to find reads with multiple nucleotides of $95 \%$ accuracy was vanishingly small. Specifically, among 500,000 reads, the lowest observed cumulative accuracy was $77 \%$. Such a result, for example, could be obtained in a sequence that has 27 "perfect" nucleotides and 5 nucleotides with a Phred $=13$ score: $(1)^{27}(0.95)^{5}=0.77$. Applying the most stringent cutoff to eliminate all reads with a Phred $<30$ yielded a library ${ }^{30} n$ in which every nucleotide had $99.9 \%$ confidence. The average confidence of the reads improved subtly from $99.2 \%$ to $99.6 \%$. The number of total reads in ${ }^{30} n$ was $30 \%$ less than number of reads in ${ }^{13} n$; that is, $\operatorname{sum}\left({ }^{30} n\right)=0.7 \operatorname{sum}\left({ }^{13} n\right)$. It was not clear whether such cutoff is an improvement or a detriment for analysis. In the next section, we examined how frequency of the members of the library changed upon application of each error cutoff.

\subsection{Example of Error Analysis: Sequence-Specific Censorship} during Phred Quality Cutoff. If errors occur by random chance, they should be uniformly distributed in all sequences. Removal of erroneous read, in that case, should be identical to sampling of the library by ${ }^{\mathrm{f}} \mathrm{Sa}$ operator, where $\mathrm{f}$ is the sampling fraction. For example, consider the removal of Phred $<13$ nucleotides from an unfiltered library (process denoted as ${ }^{1} n \rightarrow{ }^{13} n$ ). From the experiments, we know that $\operatorname{sum}\left({ }^{13} n\right)=0.9 \operatorname{sum}\left({ }^{1} n\right)$; if errors were distributed in sequences at random, the ${ }^{1} n$ and ${ }^{13} n$ vectors should be related as

$$
{ }^{13} n={ }^{0.9} \mathrm{Sa}\left({ }^{1} n\right) .
$$

The solutions should reside within a confidence interval

$$
{ }^{13} n \in\left[{ }^{\text {lo }} \mathrm{C}{ }^{\text {hi }} \mathrm{C}\right]
$$

If errors occur preferentially in specific reads, the frequency of these reads should occur beyond the confidence interval of the ${ }^{0.9} \mathrm{Sa}$. This process could be described by a diagonal matrix Bias as

$$
{ }^{13} n={ }^{0.9} \mathbf{S a}\left(\operatorname{Bias}\left({ }^{1} n\right)\right) .
$$

The elements of the diagonal matrix Bias $=\left\|\mathrm{B}_{i i}\right\|$ could be estimated as follows:

$$
\begin{aligned}
& { }^{13} n_{i} \in\left[{ }^{\mathrm{lo}} C_{i}{ }^{\mathrm{hi}} C_{i}\right], \quad \mathrm{B}_{i i}=1, \\
& { }^{13} n_{i}<{ }^{\mathrm{lo}} C_{i}, \quad \mathrm{~B}_{i i}=\frac{{ }^{13} n_{i}}{\left(0.9^{1} n_{i}\right)} .
\end{aligned}
$$

Figure 6(c) describes the representative solution of the ${ }^{0.9} \mathrm{Sa}\left({ }^{1} n\right)$ (green dots) and the confidence interval (blue lines). Supplementary Scheme S3 describes the script that calculated this interval from multiset ${ }^{1} n$, described as a plain text file PhD7-Amp-0F.txt, using 10,000 iterative calculations of ${ }^{0.9} \mathrm{Sa}\left({ }^{1} n\right)$. This calculation required $\sim 2$ hours on a desktop computer. Confidence interval was estimated as the minimum and maximum copy number found after 10,000 iterations. In this approximation of the confidence interval, for sequences with the copy number $<10$ before sampling, it was impossible to determine whether the sequence disappeared due to random sampling or due to bias. The values of Bias operator cannot be defined for these sequences and it could be assumed to be 1 (see (11)). For copy number $>10$, however, sequence-specific bias can be readily detected. We observed that the removal of Phred $<13$ reads yielded a multiset in which a large number of sequences deviated beyond the confidence interval (Figure 6(d)). Their sequences could be readily extracted by comparing the vector ${ }^{13} n$ with the vector of the lower confidence intervals ${ }^{\text {lo }} \mathrm{C}$ (see (12)). The solution of the Bias can be illustrated graphically (Figure 6(e)). Top 30 censored sequences are listed in Table S1; the other sequences can be found in the supplementary information (file PhD7Amp-0F-13F-CEN.txt).

We performed similar calculations for ${ }^{1} n \rightarrow{ }^{30} n$ and ${ }^{13} n \rightarrow{ }^{30} n$ processes. The latter process is the most interesting because ${ }^{13} n$ library has all nucleotides within acceptable confidence range (>95\%) and the distribution of cumulative quality suggested that errors, on average, do not cluster in one read (Figure 5). The ${ }^{13} n \rightarrow{ }^{30} n$ conversion eliminated $30 \%$ of the reads, and copy numbers of many sequences deviated significantly from the random sampling; these sequences are represented by green dots outside the blue confidence interval in Figure 6(h). Top 30 sequences are listed in Table S2. The censorship is not only sequence-specific, but also position-specific. In sequences that had been censored during the ${ }^{13} n \rightarrow{ }^{30} n$ process, lower quality reads clustered around 3-4 specific nucleotides (supplementary information Figure S5).

The mechanism that leads to the disappearance of censored sequences is not currently clear. We attempted to identify common motifs in censored sequences using two approaches: (i) clustering and principal component analyses based on Jukes-Cantor distance between sequences and (ii) identification of motifs using multiple unique sequence identifier software (MUSI) [17]. These approaches could not detect any property common to censored reads, which would make them significantly different from the other, noncensored reads. Still, we hypothesize that the observed 

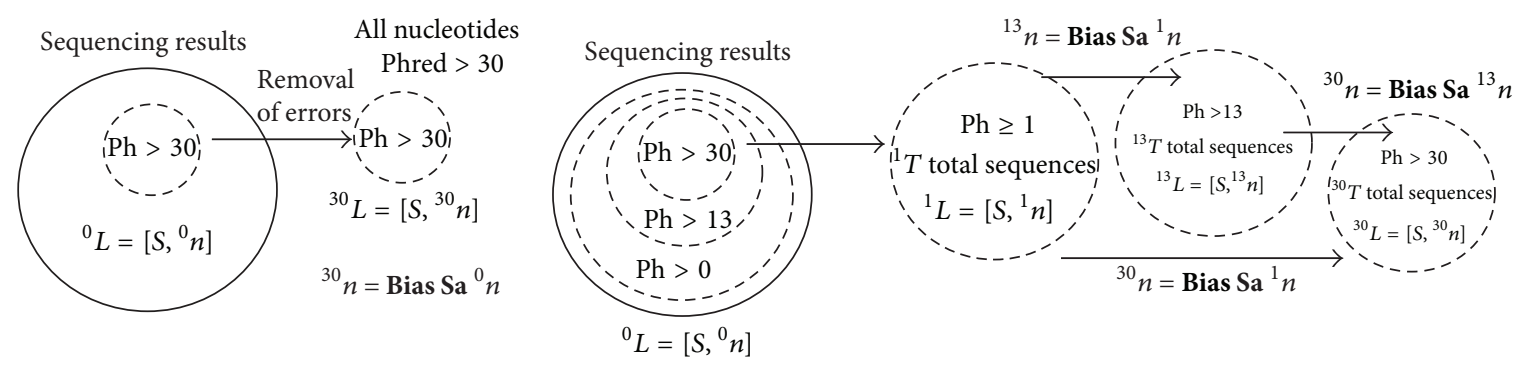

(a)

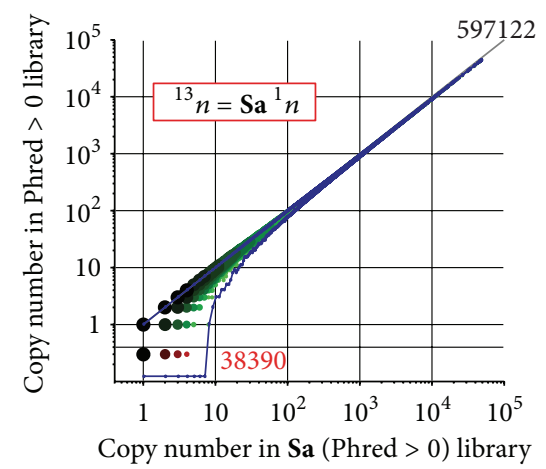

(c)

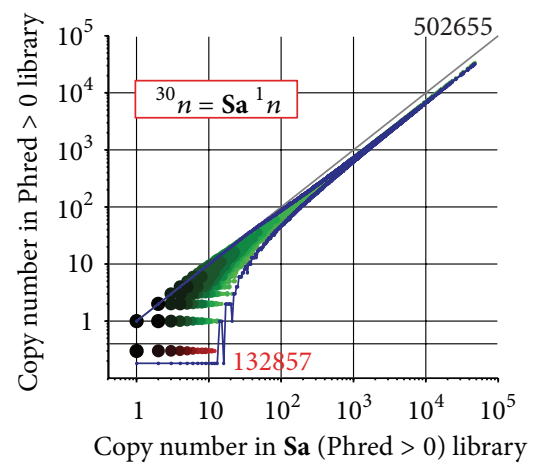

Number of data points per pixel

$\begin{array}{ll}->30000 & \cdot>100 \\ ->10000 & \cdot>30 \\ \text { - }>3000 & \cdot>10 \\ \text { - }>1000 & \cdot 1-10 \\ \text { - }>300 & \end{array}$

(f)

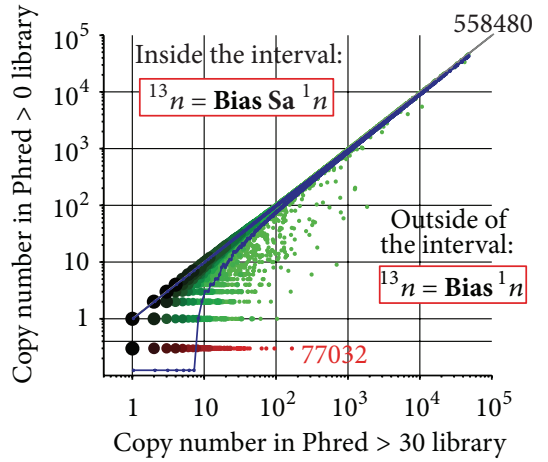

(d)

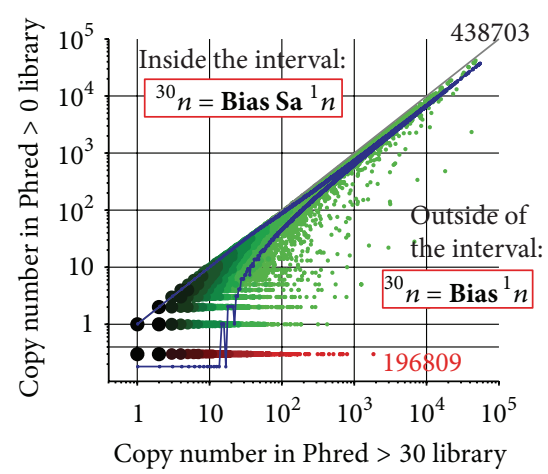

Number of data points per pixel

$\begin{array}{ll}->30000 & \cdot>100 \\ ->10000 & \cdot>30 \\ ->3000 & \cdot>10 \\ ->1000 & \cdot 1-10 \\ ->300 & \end{array}$

(g) (b)

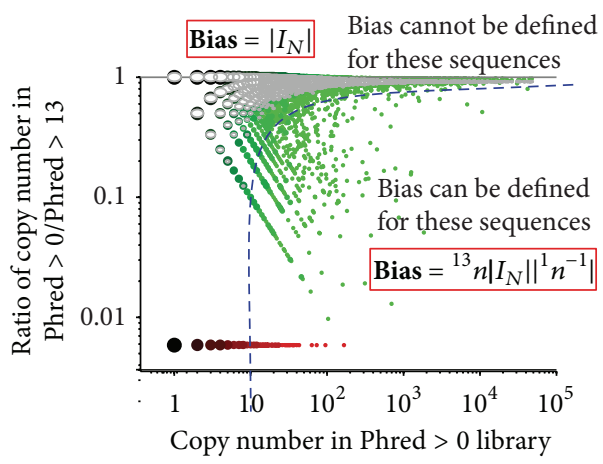

(e)

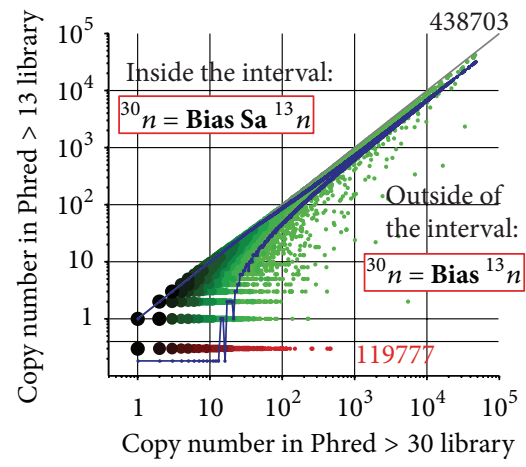

$\begin{array}{ll}\text { Number of data points per pix } \\ ->30000 & \bullet>100 \\ ->10000 & \cdot>30 \\ ->3000 & \cdot>10 \\ ->1000 & \cdot 1-10 \\ ->300 & \end{array}$

(h)

Figure 6: (a) Operator and multiset description of the error filtering procedure. Applying a Phred $>30$ cutoff to library filtered by Phred $>1$ cutoff $\left({ }^{1} n\right)$ yields a subpopulation of the library $\left({ }^{30} \mathrm{n}\right)$. If errors are sequence-independent, the ${ }^{1} \mathrm{n} \rightarrow{ }^{30} \mathrm{n}$ process should be identical to random sampling $\left({ }^{30} \mathrm{n}=\mathrm{Sa}^{1} \mathrm{n}\right)$. Any sequence-specific bias (Bias) should be detected as deviation from Sa ${ }^{1} \mathrm{n}$. (b) Progressive sampling with more stringent cutoff. (c) Theoretical Sa ${ }^{1} \mathrm{n}$ and theoretical $99.9 \%$ confidence interval (blue). (d) Observation of statistically significant deviation from Sa operator: dots beyond the blue line represent sequences prone to bias. Red dots represent sequences that disappeared after in ${ }^{1} \mathrm{n} \rightarrow{ }^{30} \mathrm{n}$ process or during $\mathbf{S a}{ }^{1} \mathrm{n}$ sampling. (e) Magnitude of the bias range from 5 to 100 -fold. (f) Bias in sampling of Phred $>30$ data from Phred $>1$ data ( $(\mathrm{f})$ is theory, $(\mathrm{g})$ is observed). (h) Bias upon sampling of Phred $>30$ data from Phred $>13$. Many sequences were lost in this sampling and this loss was statistically significant beyond the $99.9 \%$ interval. This result shows that some sequences have propensity to harbor low- and medium-quality reads. Distribution of the errors is sequence specific.

censorship represents sequence-specific errors, which occur in every time such sequence passes though the Illumina analyzer. For example, the sequences listed in Tables S1 and S2 and supplementary files were censored in five independent experiments, which were pooled and processed simultaneously in one Illumina run. Analysis of other instances of Illumina sequencing performed by other groups could help prove (or disprove) that censorship is indeed sequence-specific 
and experiment-independent. Sequence-specific censorship during Illumina analysis has been described in other publications [46]. The observations presented above suggest that reading of some sequences in phage libraries does not yield an accurate copy number. Even if these sequences were enriched due to binding, their apparent copy number in sequencing would be decreased due to sequencing bias. If the magnitude of bias is known, however, such error could be corrected. We anticipate that other biases could be calculated for these and other libraries in similar fashion. Their calculation extends beyond the scope of this paper and it will be performed in our next publication.

\section{Discussion}

3.1. Significance and Transformative Potential of LibraryWide Error Correction. In the Medicinal Chemistry field, structure-activity relationships (SAR) and pharmacophores are built using both positive and negative observations. It is the negative results that bear the most significance in these studies because they allow mapping of the range of conditions under which particular structure no longer works. For example, SAR of an R group of a ligand might be built on the following observations. A ligand binds to the target when the R group in the specific position is methyl or ethyl; changing $\mathrm{R}$ to iso-propyl and tert-butyl ablates the binding. This concludes that the R group must be a small alkyl group. An analogous situation is found in SAR of peptide ligands; the most important information from alanine scan mutagenesis is loss of function because it helps identifying the important residues. Interestingly, loss-of-binding conclusions are never applied to phage-display. The phage-display field is driven by positive results. Most publications report and follow up only on sequences enriched in the screen and consider only large copy numbers interesting. All papers focus on sequences that were found. Very few papers in phage display ask why other sequences were not found.

One of the reasons why phage display is not used for SAR-type analysis is because negative observations in phage library cannot be determined with high confidence. From a practical point of view, measuring zero with high confidence requires the largest number of observation (the highest depth of sequencing). The payoff, however, is immense: one screen with "confident zeros" could potentially yield SAR for every possible substitution of every possible amino acid. We refer to this (theoretical) possibility as "Instant SAR," and its condensed theoretical form is described in (3) or (9) and (10). This paper demonstrates that the depth of sequencing is not the only problem towards this goal. Accurate estimate of negative results requires complete characterization of the origins of errors in sequencing which yield false negative values by censoring certain sequencing. Other types of censorship, such as growth bias, should be characterized and eliminated as well. As the phage display field is currently focused on positive results, the need for optimal error corrections and recovery of erroneous reads is low. With the rise of SARtype applications in phage display, error correction will be recognized as the most significant barrier because it could lead to improper assignment of low frequencies and negative results. Improved error correction strategies could assign a lower confidence to the sequence instead of eliminating the errors and labeling them as confident zero. Proper mathematical framework, possibly similar to the one used in this paper, could be then used to carry all confidence intervals through calculations to yield reliable SAR-type data.

We note that the framework described in this paper is suitable for the analysis of the selection from libraries in which the diversity of the libraries before and after selection could be covered entirely by deep sequencing. With the current depth of sequencing, it corresponds to mediumscale libraries of $\sim 10^{6}$ random members and affinity-matured libraries that contain $\sim 10^{6}$ point mutations. We are in the process of generating these medium-scale libraries and running selection procedures that will allow us to apply and refine our framework. In the future, as technical capabilities and depth of sequencing increase, the process would be applicable to larger libraries as well.

\section{Methods}

4.1. Generation of Z-Bars and Other Visualization Techniques. Sequencing of the libraries has been described in our previous publications $[6,47]$. All data visualization in this paper was done by MATLAB scripts; raw * .eps output from MATLAB scripts subject to minor postprocessing in Adobe Illustrator to adjust fonts relative dimensions of plots. Core scripts are described in the supplementary information. Other scripts are available in our previous publication [47]. Illumina files used for the analysis can be found in the directory at http://www.chem.ualberta.ca/ derda/mathbiology/; the file ERROR_TAG_data0001.txt is an example of error-tagged reads; PhD7-Amp-xxF.txt is the library filtered with xx Phred cutoff ( $\mathrm{xx}=1,13$ and 30); file PhD7-Amp-13F-30F-CO.txt describes confidence intervals for Phred(13) to Phred(30) filtering process; other files with * ${ }^{*}$-CO.txt extension describe confidence intervals of other processes. Supplementary Figures S1-S4 and Schemes S1 and S2 describe MATLAB implementation of the Sa operator.

\section{Acknowledgments}

This work was supported by funds from the University of Alberta and Alberta Glycomics Centre.

\section{References}

[1] J. K. Scott and G. P. Smith, "Searching for peptide ligands with an epitope library," Science, vol. 249, no. 4967, pp. 386-390, 1990.

[2] G. P. Smith and V. A. Petrenko, "Phage display," Chemical Reviews, vol. 97, no. 2, pp. 391-410, 1997.

[3] A. D. Ellington and J. W. Szostak, "In vitro selection of RNA molecules that bind specific ligands," Nature, vol. 346, no. 6287, pp. 818-822, 1990.

[4] C. Tuerk and L. Gold, "Systemic evolution of ligands by exponential enrichment: RNA ligands to bacteriophage T4 DNA polymerase," Science, vol. 249, no. 4968, pp. 505-510, 1990. 
[5] E. Dias-Neto, D. N. Nunes, R. J. Giordano et al., "Nextgeneration phage display: integrating and comparing available molecular tools to enable costeffective high-throughput analysis," PLoS ONE, vol. 4, no. 12, Article ID e8338, 2009.

[6] W. L. Matochko, K. Chu, B. Jin, S. W. Lee, G. M. Whitesides, and R. Derda, "Deep sequencing analysis of phage libraries using Illumina platform," Methods, vol. 58, pp. 47-55, 2012.

[7] A. Ernst, D. Gfeller, Z. Kan et al., "Coevolution of PDZ domainligand interactions analyzed by high-throughput phage display and deep sequencing," Molecular BioSystems, vol. 6, no. 10, pp. 1782-1790, 2010.

[8] G. V. Kupakuwana, J. E. Crill, M. P. McPike, and P. N. Borer, "Acyclic identification of aptamers for human alpha-thrombin using over-represented libraries and deep sequencing," PLoS ONE, vol. 6, no. 5, Article ID e19395, 2011.

[9] P. A. C. 'T Hoen, S. M. G. Jirka, B. R. Ten Broeke et al., "Phage display screening without repetitious selection rounds," Analytical Biochemistry, vol. 421, no. 2, pp. 622-631, 2012.

[10] H. Zhang, A. Torkamani, T. M. Jones, D. I. Ruiz, J. Pons, and R. A. Lerner, "Phenotype-information-phenotype cycle for deconvolution of combinatorial antibody libraries selected against complex systems," Proceedings of the National Academy of Sciences of the United States of America, vol. 108, no. 33, pp. 13456-13461, 2011.

[11] R. Derda, S. K. Y. Tang, S. C. Li, S. Ng, W. Matochko, and M. R. Jafari, "Diversity of phage-displayed libraries of peptides during panning and amplification," Molecules, vol. 16, no. 2, pp. 17761803, 2011.

[12] R. W. Hamming, "Error detecting and error correcting codes," Bell System Technical Journal, vol. 29, pp. 147-160, 1950.

[13] D. J. Rodi, A. S. Soares, and L. Makowski, "Quantitative assessment of peptide sequence diversity in M13 combinatorial peptide phage display libraries," Journal of Molecular Biology, vol. 322, no. 5, pp. 1039-1052, 2002.

[14] L. Makowski, "Quantitative analysis of peptide libraries," in Phage Nanobiotechnology, chapter 3, 2011.

[15] C. E. Shannon, "A mathematical theory of communication," Bell System Technical Journal, vol. 27, pp. 379-423, 1948.

[16] U. Ravn, F. Gueneau, L. Baerlocher et al., "By-passing in vitro screening: next generation sequencing technologies applied to antibody display and in silico candidate selection," Nucleic Acids Research, vol. 38, no. 21, 2010.

[17] T. Kim, M. S. Tyndel, H. Huang et al., "MUSI: an integrated system for identifying multiple specificity from very large peptide or nucleic acid data sets," Nucleic Acids Research, vol. 40, no. 6, article e47, 2012.

[18] J. A. Weinstein, N. Jiang, R. A. White III, D. S. Fisher, and S. R. Quake, "High-throughput sequencing of the zebrafish antibody repertoire," Science, vol. 324, no. 5928, pp. 807-810, 2009.

[19] B. J. DeKosky, G. C. Ippolito, R. P. Deschner et al. et al., "Highthroughput sequencing of the paired human immunoglobulin heavy and light chain repertoire," Nature Biotechnology, vol. 31, pp. 166-169, 2013.

[20] B. Levitan, "Stochastic modeling and optimization of phage display," Journal of Molecular Biology, vol. 277, no. 4, pp. 893916, 1998.

[21] C. S. Riesenfeld, P. D. Schloss, and J. Handelsman, "Metagenomics: genomic analysis of microbial communities," Annual Review of Genetics, vol. 38, pp. 525-552, 2004.

[22] M. L. Sogin, H. G. Morrison, J. A. Huber et al., "Microbial diversity in the deep sea and the underexplored 'rare biosphere,"
Proceedings of the National Academy of Sciences of the United States of America, vol. 103, no. 32, pp. 12115-12120, 2006.

[23] F. Bäckhed, R. E. Ley, J. L. Sonnenburg, D. A. Peterson, and J. I. Gordon, "Host-bacterial mutualism in the human intestine," Science, vol. 307, no. 5717, pp. 1915-1920, 2005.

[24] N. Beerenwinkel and O. Zagordi, "Ultra-deep sequencing for the analysis of viral populations," Current Opinion in Virology, vol. 1, no. 5, pp. 413-418, 2011.

[25] J. A. Huber, D. B. Mark Welch, H. G. Morrison et al., "Microbial population structures in the deep marine biosphere," Science, vol. 318 , no. 5847, pp. 97-100, 2007.

[26] A. Wilm, P. P. K. Aw, D. Bertrand et al., "LoFreq: a sequencequality aware, ultra-sensitive variant caller for uncovering cellpopulation heterogeneity from high-throughput sequencing datasets," Nucleic Acids Research, vol. 40, pp. 11189-11201, 2012.

[27] P. J. Turnbaugh, C. Quince, J. J. Faith et al., "Organismal, genetic, and transcriptional variation in the deeply sequenced gut microbiomes of identical twins," Proceedings of the National Academy of Sciences of the United States of America, vol. 107, no. 16, pp. 7503-7508, 2010.

[28] C. Quince, A. Lanzén, T. P. Curtis et al., "Accurate determination of microbial diversity from 454 pyrosequencing data," Nature Methods, vol. 6, no. 9, pp. 639-641, 2009.

[29] S. J. Watson, M. R. A. Welkers, D. P. Depledge et al., "Viral population analysis and minority-variant detection using short read next-generation sequencing," Philosophical Transactions of the Royal Society B, vol. 368, no. 1614, Article ID 20120205, 2013.

[30] D. R. Bentley, S. Balasubramanian, H. P. Swerdlow et al. et al., "Accurate whole human genome sequencing using reversible terminator chemistry," Nature, vol. 456, pp. 53-59, 2008.

[31] K. Nakamura, T. Oshima, T. Morimoto et al., "Sequence-specific error profile of Illumina sequencers," Nucleic Acids Research, vol. 39, no. 13, 2011.

[32] M. A. Quail, I. Kozarewa, F. Smith et al., "A large genome center's improvements to the Illumina sequencing system," Nature Methods, vol. 5, no. 12, pp. 1005-1010, 2008.

[33] O. Zagordi, R. Klein, M. Däumer, and N. Beerenwinkel, "Error correction of next-generation sequencing data and reliable estimation of HIV quasispecies," Nucleic Acids Research, vol. 38, no. 21, pp. 7400-7409, 2010.

[34] M. W. Schmitt, S. R. Kennedy, J. J. Salk, E. J. Fox, J. B. Hiatt, and L. A. Loeb, "Detection of ultra-rare mutations by nextgeneration sequencing," Proceedings of the National Academy of Sciences of the United States of America, vol. 109, pp. 1450814513, 2012.

[35] A. Syropoulos, "Mathematics of multisets," in Proceedings of the Workshop on Multiset Processing: Multiset Processing, Mathematical, Computer Science, and Molecular Computing Points of View, pp. 347-358, Springer, 2001.

[36] W. Arap, M. G. Kolonin, M. Trepel et al., "Steps toward mapping the human vasculature by phage display," Nature Medicine, vol. 8, no. 2, pp. 121-127, 2002.

[37] S. Blond-Elguindi, S. E. Cwirla, W. J. Dower et al., "Affinity panning of a library of peptides displayed on bacteriophages reveals the binding specificity of BiP," Cell, vol. 75, no. 4, pp. 717728, 1993.

[38] S. Cox, E. Rosten, J. Monypenny et al., "Bayesian localization microscopy reveals nanoscale podosome dynamics," Nature Methods, vol. 9, no. 2, pp. 195-200, 2012.

[39] E. Rosten, G. E. Jones, and S. Cox, "Image plug-in for Bayesian analysis of blinking and bleaching," Nature Methods, vol. 10, pp. 97-98, 2013. 
[40] W. L. Matochko, S. Ng, M. R. Jafari, J. Romaniuk, S. K. Y. Tang, and R. Derda, "Uniform amplification of phage display libraries in monodisperse emulsions," Methods, vol. 58, pp. 18-27, 2012.

[41] E. A. Peters, P. J. Schatz, S. S. Johnson, and W. J. Dower, "Membrane insertion defects caused by positive charges in the early mature region of protein pIII of filamentous phage fd can be corrected by prlA suppressors," Journal of Bacteriology, vol. 176, no. 14, pp. 4296-4305, 1994.

[42] L. A. Brammer, B. Bolduc, J. L. Kass, K. M. Felice, C. J. Noren, and M. F. Hall, "A target-unrelated peptide in an M13 phage display library traced to an advantageous mutation in the gene II ribosome-binding site," Analytical Biochemistry, vol. 373, no. 1, pp. 88-98, 2008.

[43] G. A. Kuzmicheva, P. K. Jayanna, I. B. Sorokulova, and V. A. Petrenko, "Diversity and censoring of landscape phage libraries," Protein Engineering, Design and Selection, vol. 22, no. 1, pp. 9-18, 2009.

[44] D. R. Wilson and B. B. Finlay, "Phage display: applications, innovations, and issues in phage and host biology," Canadian Journal of Microbiology, vol. 44, no. 4, pp. 313-329, 1998.

[45] R. Derda, S. K. Y. Tang, and G. M. Whitesides, "Uniform amplification of phage with different growth characteristics in individual compartments consisting of monodisperse droplets," Angewandte Chemie, vol. 49, no. 31, pp. 5301-5304, 2010.

[46] J. C. Dohm, C. Lottaz, T. Borodina, and H. Himmelbauer, "Substantial biases in ultra-short read data sets from highthroughput DNA sequencing," Nucleic Acids Research, vol. 36, no. 16, article e105, 2008.

[47] W. L. Matochko, S. C. Li, S. K. Y. Tang, and R. Derda, "Prospective identification of parasitic sequences in phage display screens," Nucleic Acids Research, 2013. 


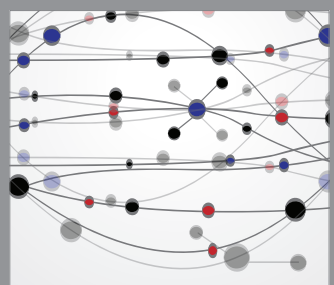

The Scientific World Journal
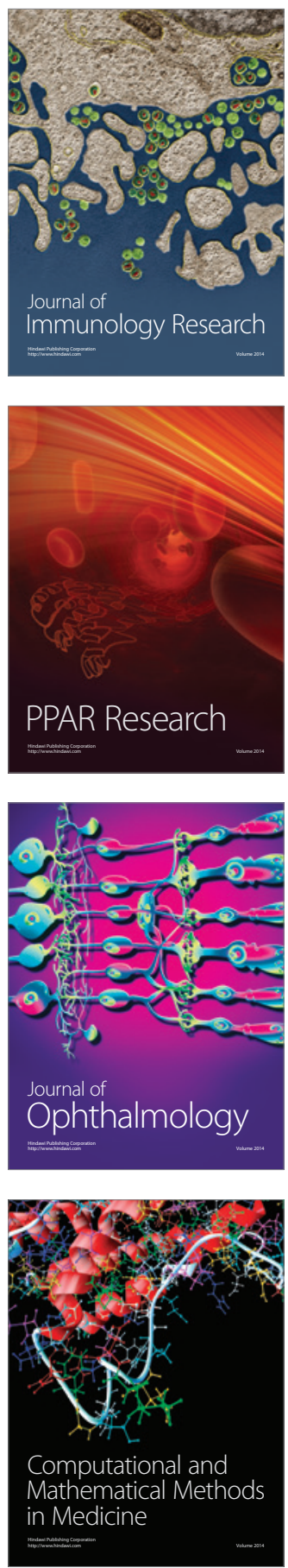

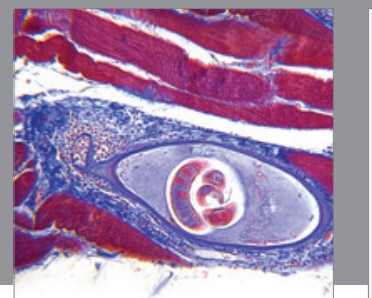

Gastroenterology

Research and Practice
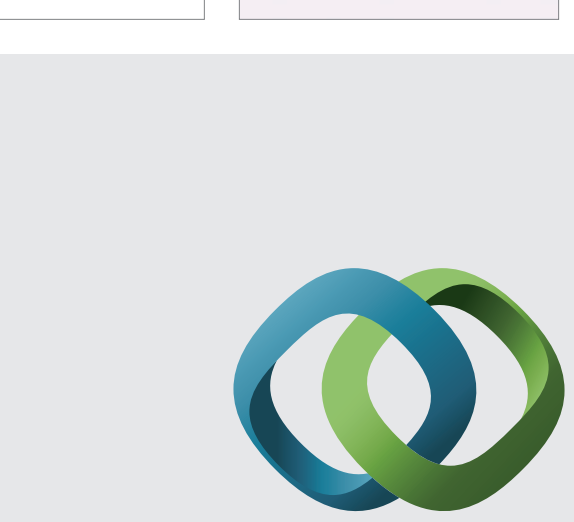

\section{Hindawi}

Submit your manuscripts at

http://www.hindawi.com
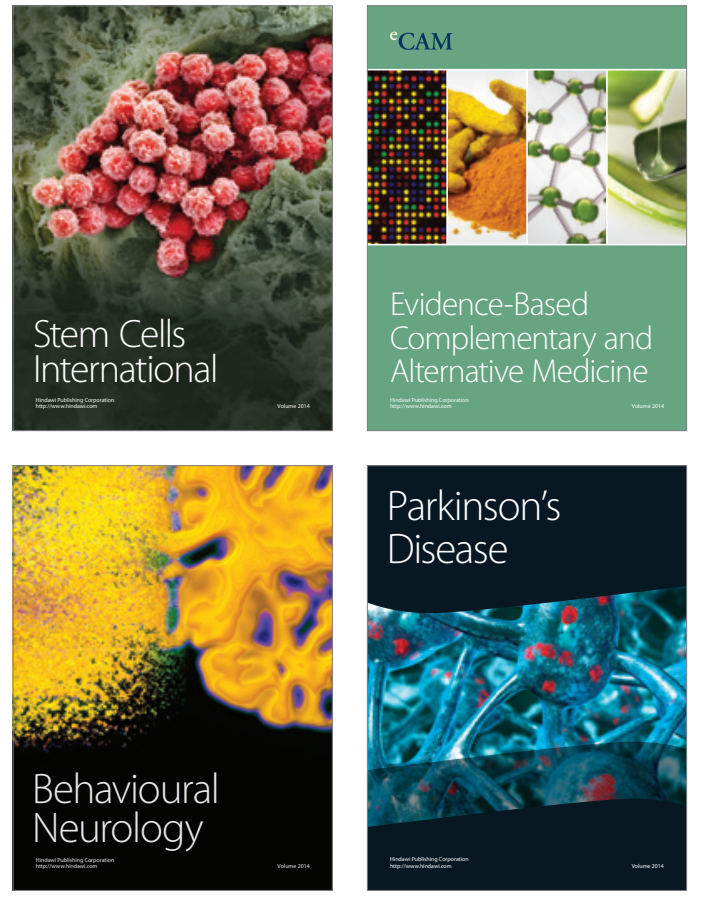
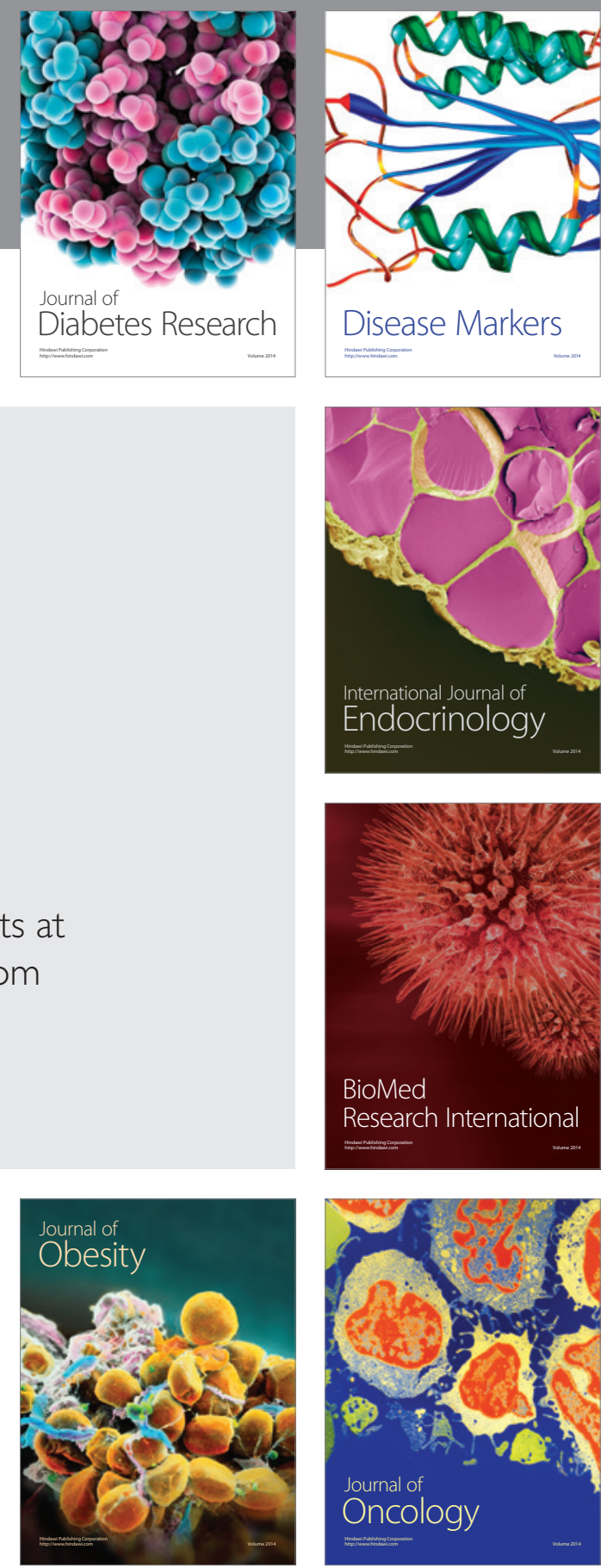

Disease Markers
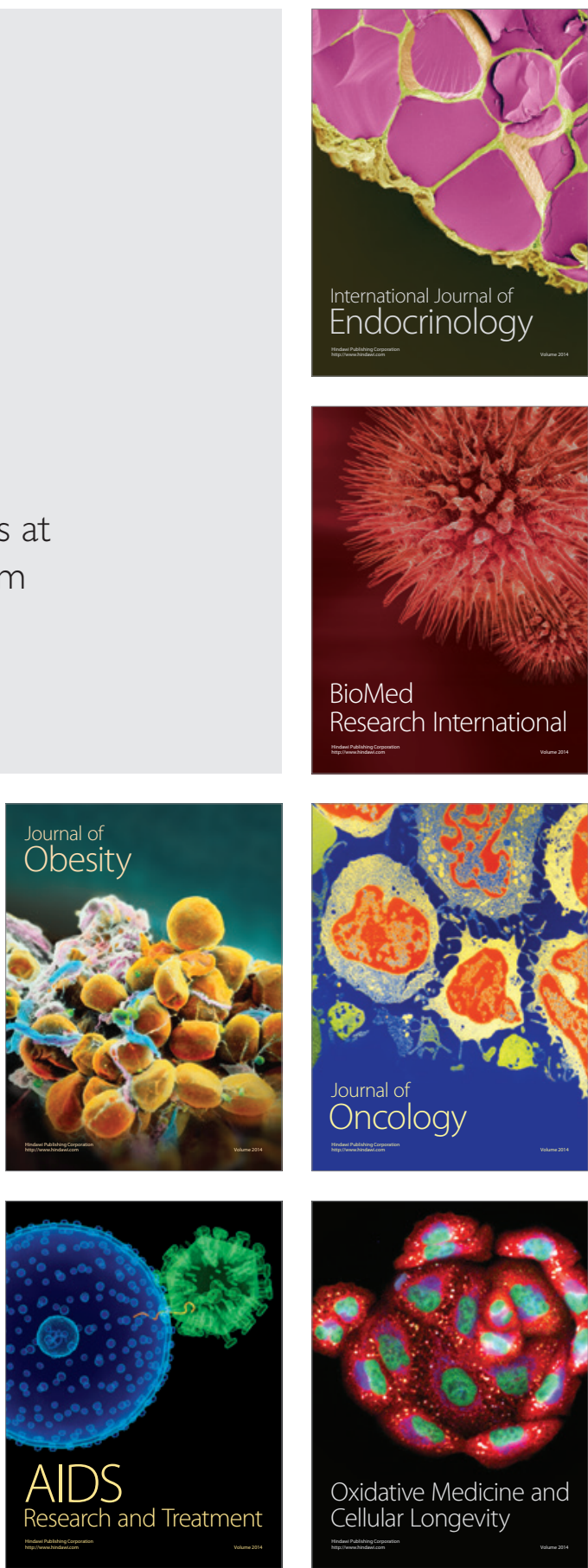